\title{
DYNAMIC MODELING OF A MOMENT EXCHANGE UNICYCLE ROBOT
}

\author{
S. Langius \\ Department of Mechanical Engineering, University of Twente, Enschede, 7500 AE, The Netherlands \\ s.langius@student.utwente.nl \\ R. A. de Callafon \\ Department of Mechanical and Aerospace Engineering, UCSD, 9500 Gilman Drive, La Jolla, CA 92093-0411, U.S.A. \\ callafon@ucsd.edu
}

\begin{abstract}
Keywords: Unicycle, Robotics, Modeling.
Abstract: In this paper a three dimensional non-linear model has been derived to describe the dynamics of an unstable moment exchange unicycle robot. The robot uses a driving wheel to provide stabilization in longitudinal direction, while a second moment exchange wheel with a large inertia is used for stabilization in lateral direction. For validation purposes, the resulting equations of motion are compared independently against the simulation results of a finite element package called SPACAR. The model includes the coupling between the lateral and longitudinal motion, which makes it possible to control the yaw angle and the model can be used to design a stabilizing feedback controller.
\end{abstract}

\section{INTRODUCTION}

Using basic principles of kinematics and dynamics, dynamic models of robotic systems can be derived independently by hand or by an automated computer program to ensure cross model validity. This paper illustrates this approach on an inherently unstable Moment Exchange Unicycle Robot (MEUR) depicted in Figure 1 that requires stabilization in both a lateral and longitudinal direction. Using Newtonian mechanics and a finite element package called SPACAR, three dimensional non-linear models that incorporates the coupling between the lateral and longitudinal motion of the MEUR are derived. The resulting dynamic model captures the dynamics of a wide variety of moment exchange unicycle robots and can be used to design stabilizing feedback control algorithms.

Earlier work on dynamic modeling of robotic unicycles can be distinguished by their mechanism to achieve lateral stabilization (van Pommeren, 2007). An inertia wheel moving in the horizontal plane for stabilization is used in many earlier applications (Schoonwinkel, 1987; Vos and Flotow, 1990; Naveh et al., 1999). This approach is comparable to the twisting torso motion of a real unicyclist (Ohsaki et al., 2008; Sheng and Yamafuji, 1997). The gyroscopic effect of two inertias spinning in opposite di- rection in the horizontal plane is used in (Zenkov et al., 1999). A pendulum moving in the vertical plane perpendicular to that of the driving wheel is used in (Dao and Liu, 2005) and an inverted pendulum in the same plane is used in (Nakajima et al., 1997) where lateral stabilization is further improved by a barrel shaped wheel. The work of (Au and Xu, 1999) makes use of the gyroscopic effect for stabilization.

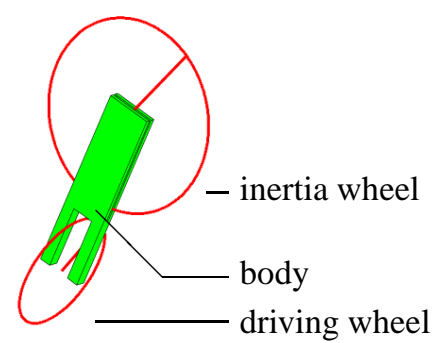

Figure 1: Schematic drawing of a Moment Exchange Unicycle Robot (MEUR).

The three dimensional model of the MEUR derived in this paper includes the coupling between the lateral and longitudinal motion, which makes it possible to control the yaw angle similar as in (Majima et al., 2006). For validation purposes of the dynamic model, the simulation results obtained from the equations of motion are compared independently 
against the simulation results of a finite element package called SPACAR (Aarts et al., 2008).

\section{EQUATIONS OF MOTION}

\subsection{Model Structure and Orientation}

The configuration and position of the the MEUR in Figure 1 can be described by six independent coordinates displayed in Table 1. The six coordinates do not include the pitch angle of the inertia wheel, as we assume that the inertia wheel is simply replaced by a moment acting on the body. This is a simplification of the problem, discarding the quadratic velocity terms caused by the (small) pitch velocity of the inertia wheel. Figure 2 shows all the transformations needed to apply Newtonian mechanics, where Table 2 describes all the coordinate systems involved.

Table 1: Definition of independent coordinates.

\begin{tabular}{|llll|}
\hline & description & applies to & axis \\
\hline$\psi$ & Yaw angle & wheel \& body & $a_{3}$ \\
$\gamma$ & Roll angle & wheel \& body & $b_{1}$ \\
$\varphi$ & Body pitch angle & wheel \& body & $c_{2}$ \\
$\theta$ & Wheel pitch angle & wheel & $d_{2}$ \\
$X$ & X-position point Q & wheel \& body & $a_{1}$ \\
$Y$ & y-position point Q & wheel \& body & $a_{2}$ \\
\hline
\end{tabular}

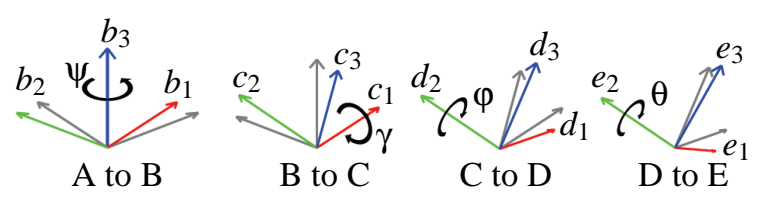

Figure 2: All coordinate system transformations.

Table 2: Definition of the coordinate systems (CS).

\begin{tabular}{|lll|}
\hline CS & description & unit vectors \\
\hline A & Inertial CS & $a_{1}, a_{2}, a_{3}$ \\
B & Contact force CS & $b_{1}, b_{2}, b_{3}$ \\
C & No-slip constraint CS & $c_{1}, c_{2}, c_{3}$ \\
D & Body CS & $d_{1}, d_{2}, d_{3}$ \\
E & Wheel CS & $e_{1}, e_{2}, e_{3}$ \\
\hline
\end{tabular}

The inertial coordinate system will be used to apply Newton's second law of motion, since this law is only valid when observing translational accelerations from this coordinate system. The contact force coordinate system is used to define the forces acting in the contact point. The no-slip constraint coordinate system is used for the no-slip constraint since the contact point is a stationary point only in this coordinate system. The body and wheel coordinate systems are used to apply Euler's equations, since their rotational inertias remain constant in these coordinate systems (Hughes, 1986).

The transformations $\underline{B}=\underline{A} \theta_{B A}, \underline{C}=\underline{B} \theta_{C B}, \underline{D}=$ $\underline{C} \theta_{D C}$ and $\underline{E}=\underline{C} \theta_{E C}$ between the different coordinate systems is captured by the rotation matrices

$$
\begin{gathered}
\theta_{B A}=\left[\begin{array}{ccc}
C_{\psi} & -S_{\psi} & 0 \\
S_{\psi} & C_{\psi} & 0 \\
0 & 0 & 1
\end{array}\right], \theta_{C B}=\left[\begin{array}{ccc}
1 & 0 & 0 \\
0 & C_{\gamma} & -S_{\gamma} \\
0 & S_{\gamma} & C_{\gamma}
\end{array}\right] \\
\theta_{D C}=\left[\begin{array}{ccc}
C_{\varphi} & 0 & S_{\varphi} \\
0 & 1 & 0 \\
-S_{\varphi} & 0 & C_{\varphi}
\end{array}\right], \theta_{E C}=\left[\begin{array}{ccc}
C_{\theta+\varphi} & 0 & S_{\theta+\varphi} \\
0 & 1 & 0 \\
-S_{\theta+\varphi} & 0 & C_{\theta+\varphi}
\end{array}\right]
\end{gathered}
$$

where the $\cos (x)$ and $\sin (x)$ terms in the rotation matrices are shortened to respectively $C_{x}$ and $S_{x}$. For dynamic analysis, the derivatives of the rotation matrices are given by $\dot{\theta}_{B A}=\theta_{B A} \tilde{\omega}_{B A}, \dot{\theta}_{C B}=\theta_{C B} \tilde{\omega}_{C B}$, $\dot{\theta}_{D C}=\theta_{D C} \tilde{\omega}_{D C}$ and $\dot{\theta}_{E C}=\theta_{E C} \tilde{\omega}_{E C}$ where

$$
\begin{aligned}
& \tilde{\omega}_{B A}=\left[\begin{array}{ccc}
0 & -\dot{\psi} & 0 \\
\dot{\psi} & 0 & 0 \\
0 & 0 & 0
\end{array}\right], \tilde{\omega}_{C B}=\left[\begin{array}{ccc}
0 & 0 & 0 \\
0 & 0 & -\dot{\gamma} \\
0 & \dot{\gamma} & 0
\end{array}\right] \\
& \tilde{\omega}_{D C}=\left[\begin{array}{ccc}
0 & 0 & \dot{\varphi} \\
0 & 0 & 0 \\
-\dot{\varphi} & 0 & 0
\end{array}\right], \tilde{\omega}_{E C}=\left[\begin{array}{ccc}
0 & 0 & \dot{\alpha} \\
0 & 0 & 0 \\
-\dot{\alpha} & 0 & 0
\end{array}\right]
\end{aligned}
$$

where $\dot{\alpha}=\dot{\theta}+\dot{\varphi}$.

\subsection{Wheel Equations}

The angular velocity vector of the wheel $\underline{\omega}$ can be expressed as the sum of all individual angular velocities each defined in their own coordinate system. When all these angular velocities are transformed to coordinate system $\mathrm{C}$, leads to an expression

$$
\underline{\omega}_{C}=\theta_{C B}{ }^{T}\left\{\begin{array}{c}
0 \\
0 \\
\dot{\psi}
\end{array}\right\}+\left\{\begin{array}{c}
\dot{\gamma} \\
0 \\
0
\end{array}\right\}+\theta_{E C}\left\{\begin{array}{c}
0 \\
\dot{\theta}+\dot{\varphi} \\
0
\end{array}\right\}
$$

and correspondingly, $\tilde{\omega}_{C}$ is a matrix composed of the elements of vector $\underline{\omega}_{C}$ given by

$$
\tilde{\omega}_{C}=\left[\begin{array}{ccc}
0 & -\underline{\omega}_{C 3} & \underline{\omega}_{C 2} \\
\underline{\omega}_{C 3} & 0 & -\underline{\omega}_{C 1} \\
-\underline{\omega}_{C 2} & \underline{\omega}_{C 1} & 0
\end{array}\right]
$$

Vector $\underline{r}_{Q P}$ is shown in Figure 3 and is the vector going from point $P$ (the contact point of the wheel) to point $\mathrm{Q}$ (the center of the wheel), pointing in the $c_{3}$ direction and its length is equal to radius of the wheel $\underline{r}_{Q P, C}=\left\{\begin{array}{lll}0 & 0 & R\end{array}\right\}^{T}$. Since no slip is assumed, the velocity at the contact point satisfies $\underline{v}_{P, C}=\underline{0}$. The velocity of the center of the wheel expressed in coordinate system $\mathrm{C}$ is equal to the velocity of the contact point plus the relative velocity due to rotation

$$
\underline{v}_{Q, C}=\underline{v}_{P, C}+\underline{\omega}_{C} \times \underline{r}_{Q P, C}=\tilde{\omega}_{C} \underline{r}_{Q P, C}
$$


following (Kolve, 1993) to describe the derivative of the rotation matrices and replacing the cross product by a matrix multiplication. The same velocity can be transformed to coordinate system A, which is the velocity $\underline{v}_{Q, A}$ with respect to the fixed world given by

$$
\underline{v}_{Q, A}=\theta_{B A} \theta_{C B}\left(\underline{v}_{P, C}+\tilde{\omega}_{C} \underline{r}_{Q P, C}\right)
$$

Writing out the right hand side of (3) leads to the differential equations for the components of $\underline{v}_{Q, A}$ :

$$
\begin{aligned}
& \dot{x}=R\left(\left(\dot{\theta}+\dot{\varphi}+\dot{\psi} S_{\gamma}\right) C_{\psi}+\dot{\gamma} C_{\gamma} S_{\psi}\right) \\
& \dot{y}=R\left(\left(\dot{\theta}+\dot{\varphi}+\dot{\psi} S_{\gamma}\right) S_{\psi}-\dot{\gamma} C_{\gamma} C_{\psi}\right) \\
& \dot{z}=-R \dot{\gamma} S_{\gamma}
\end{aligned}
$$

Solving (4) and (5) for $x$ and $y$ results in the solution for the first 2 independent coordinates. This can only be done numerically since these equations are nonintegrable. The solution for $z=R C_{\gamma}$ can be obtained from the analytical solution of the integral of (6).

To find an expression for the acceleration of point $\mathrm{Q}$, first (1) will be differentiated to obtain

$$
\begin{gathered}
\underline{\omega}_{C}=-\tilde{\omega}_{C B} \theta_{C B}{ }^{T}\left\{\begin{array}{c}
0 \\
0 \\
\dot{\psi}
\end{array}\right\}+\theta_{C B}{ }^{T}\left\{\begin{array}{c}
0 \\
0 \\
\ddot{\psi}
\end{array}\right\}+\left\{\begin{array}{l}
\ddot{\gamma} \\
0 \\
0
\end{array}\right\} \cdots \\
\cdots+\theta_{E C} \tilde{\omega}_{E C}\left\{\begin{array}{c}
0 \\
\dot{\theta}+\dot{\varphi} \\
0
\end{array}\right\}+\theta_{E C}\left\{\begin{array}{c}
0 \\
\ddot{\theta}+\ddot{\varphi} \\
0
\end{array}\right\}
\end{gathered}
$$

and again, $\dot{\tilde{\omega}}_{C}$ is a matrix composed of the elements of vector $\underline{\dot{\omega}}_{C}$ given by

$$
\dot{\tilde{\omega}}_{C}=\left[\begin{array}{ccc}
0 & -\underline{\dot{\omega}}_{C 3} & \underline{\dot{\omega}}_{C 2} \\
\underline{\dot{\omega}}_{C 3} & 0 & -\underline{\dot{\omega}}_{C 1} \\
-\underline{\dot{\omega}}_{C 2} & \underline{\dot{\omega}}_{C 1} & 0
\end{array}\right]
$$

Differentiating (3) now leads to an expression for the acceleration of point $\mathrm{Q}$ given by

$$
\dot{\underline{v}}_{Q, A}=\theta_{B A}\left(\tilde{\omega}_{B A} \theta_{C B} \tilde{\omega}_{C}+\theta_{C B} \tilde{\omega}_{C B} \tilde{\omega}_{C}+\theta_{C B} \dot{\tilde{\omega}}_{C}\right) \underline{r}_{Q P, C}
$$

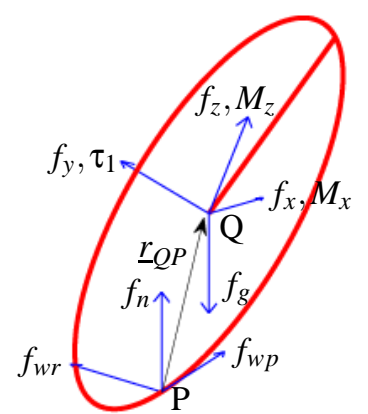

Figure 3: Forces and moments acting on the wheel.

The gravity force vector $\underline{F}_{G, B}$ does not move with any of the angles and since its direction is unaffected by rotation matrix $\theta_{B A}$ it can be defined in the coordinate system B via $\underline{F}_{G, B}=\left\{\begin{array}{ll}0 & 0-m_{w} g\end{array}\right\}_{B}^{T}$ because of its simplicity later on. The contact force vector $\underline{F}_{C, B}$ is chosen to be defined in coordinate system $\mathrm{B}$, so that it always rotates with the yaw angle $\psi$ but stays in the same plane as the ground on which the wheel moves. In this way $f_{w p}$ always points in driving direction and $f_{w r}$ points in perpendicular direction. The third element working on the same point is the normal force $f_{n}$ creating $\underline{F}_{C, B}=\left\{f_{w p} f_{w r} f_{n}\right\}_{B}^{T}$. Finally, the reaction force vector $\underline{F}_{R, D}=\left\{f_{x} f_{y} f_{z}\right\}_{D}$ is chosen to be defined in coordinate system $\mathrm{D}$, so that it moves with the body.

Newton's second law of motion yields

$$
\sum \underline{F}_{A}=m_{w} \underline{\dot{v}}_{Q, A}
$$

where the left hand side is the sum of previously mentioned forces, transformed to the inertial coordinate system $A$, given by

$$
\sum \underline{F}_{A}=\theta_{B A}\left(\underline{F}_{C, B}+\underline{F}_{G, B}+\theta_{C B} \theta_{D C} \underline{F}_{R, D}\right)
$$

and leads to an expression for the unknown contact force vector

$$
\underline{F}_{C, B}=\theta_{B A}{ }^{T} m_{w} \underline{\dot{\dot{x}}}_{Q, A}-F_{G, B}-\theta_{C B} \theta_{D C} F_{R, D}
$$

The reaction moment vector $\underline{M}_{R, D}$ moves with the body, just like the reaction force vector, and is therefore defined in coordinate system $\mathrm{D}$. The second element of $\underline{M}_{R, D}$ is equal to the torque applied by the motor between the driving wheel and body leading to $\underline{M}_{R, D}=\left\{M_{x} \tau_{1} M_{z}\right\}_{D}$. Since the wheel is rotation symmetric about the $c_{2}$-axis, the pitch angles have no influence on the inertias and Euler's equation

$$
\sum \underline{M}=\underline{\dot{h}}
$$

applies in coordinate system $\mathrm{C}$. The angular momentum is expressed in coordinate system $\mathrm{C}$ by

$$
\underline{h}=J \cdot \underline{\omega}=\left(\underline{C}_{C} \underline{C}^{T}\right) \cdot\left(\underline{C \omega}_{C}\right)=\underline{C}_{C} \underline{\omega}_{C}
$$

where

$$
J_{C}=\left[\begin{array}{ccc}
J_{x x} & 0 & 0 \\
0 & J_{y y} & 0 \\
0 & 0 & J_{z z}
\end{array}\right]
$$

The derivative of $\underline{h}$ can then be written as

$$
\begin{aligned}
\underline{\dot{h}} & =\underline{\dot{C}} J_{C} \underline{\omega}_{C}+\underline{C}_{C} \underline{\omega}_{C}+\underline{C}_{C} \underline{\dot{\omega}}_{C} \\
& =\underline{C}\left(\tilde{\omega}_{C A} J_{C} \underline{\omega}_{C}+J_{C} \underline{\dot{\omega}}_{C}\right)
\end{aligned}
$$

and substituting (9) into (8) with $\sum \underline{M}=\underline{C} \sum \underline{M}_{C}$ yields

$$
\sum \underline{M}_{C}=\tilde{\omega}_{C A} J_{C} \underline{\omega}_{C}+J_{C} \underline{\dot{\omega}}_{C}
$$

Finally, the left hand side of (10) can be written as

$$
\sum \underline{M}_{C}=\theta_{D C} \underline{M}_{R, D}-\underline{\tilde{r}}_{Q P, C} \theta_{C B}{ }^{T} \underline{F}_{C, B}
$$


where

$$
\underline{\tilde{r}}_{Q P, C}=\left[\begin{array}{ccc}
0 & -\underline{r}_{Q P, C 3} & \underline{r}_{Q P, C 2} \\
\underline{r}_{Q P, C 3} & 0 & -\underline{r}_{Q P, C 1} \\
\underline{-}_{Q P, C 2} & \underline{r}_{Q P, C 1} & 0
\end{array}\right]
$$

As a final note it can be observed from Figure 3 that the only force vector creating a moment on point $\mathrm{Q}$ is the contact force vector and the arm for this moment is in opposite direction of $\underline{r}_{Q P, C}$. For the final moment balance, the reacting force and moment vector have to be transformed to coordinate system $\mathrm{C}$ first.

\subsection{Body Equations}

Figure 4 shows the forces and moments acting on the body, where $\underline{r}_{R Q, D}=\left\{\begin{array}{lll}0 & 0 & d_{R Q}\end{array}\right\}_{D}$ is the vector going from point $\mathrm{Q}$ to $\mathrm{R}$ and the components of $\underline{F}_{R}$ and $\underline{M}_{R}$ are defined in positive direction on the wheel and thus work in opposite direction on the body. The gravity force vector $\underline{F}_{G B, A}=\left\{\begin{array}{ll}0 & 0-m_{b} g\end{array}\right\}_{A}^{T}$ acts on point R, the center of gravity for the body and inertia wheel together. The inertia wheel is simply replaced by a moment, $\underline{M}_{I, D}=\left\{\begin{array}{llll}\tau_{2} & 0 & 0\end{array}\right\}_{D}$, where the assumption is made that low inertial wheel velocity allows moments caused by quadratic velocity terms to be discarded.

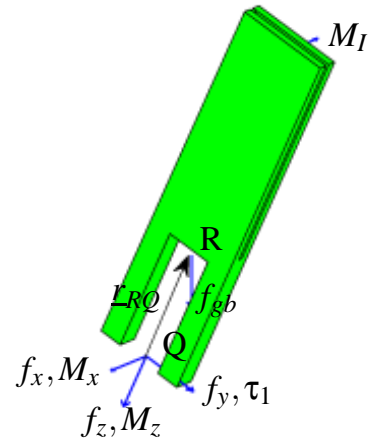

Figure 4: Forces and moments acting on the body.

The angular velocity of the body is equal to that of the wheel

$$
\underline{\omega}_{b, C}=\theta_{C B}{ }^{T}\left\{\begin{array}{c}
0 \\
0 \\
\dot{\psi}
\end{array}\right\}+\left\{\begin{array}{c}
\dot{\gamma} \\
0 \\
0
\end{array}\right\}+\theta_{E C}\left\{\begin{array}{c}
0 \\
\dot{\varphi} \\
0
\end{array}\right\}
$$

however it does not rotate with angle $\theta$. The velocity of point $\mathrm{R}$ is equal to the velocity of point $\mathrm{Q}$ plus the relative velocity due to rotation

$$
\underline{v}_{R, A}=\underline{v}_{Q, A}+\tilde{\omega}_{b, A} \underline{r}_{R Q, A}
$$

where $\tilde{\omega}_{b, A}$ and $\underline{r}_{R Q, A}$ in (12) can be expressed by the known equations for $\tilde{\omega}_{b, C}$ and $\underline{r}_{R Q, D}$ given by

$$
\begin{aligned}
& \tilde{\omega}_{b, A}=\theta_{B A} \theta_{C B} \tilde{\omega}_{b, C} \theta_{C B}^{T} \theta_{B A}^{T} \\
& \underline{r}_{R Q, A}=\theta_{B A} \theta_{C B} \theta_{D C} \underline{r}_{R Q, D}
\end{aligned}
$$

and substitution of (13) and (14) into (12) yields

$$
\underline{v}_{R, A}=\underline{v}_{Q, A}+\theta_{B A} \theta_{C B} \tilde{\omega}_{b, C} \theta_{D C} \underline{r}_{R Q, D}
$$

Finally, differentiating the right hand side of (15) yields

$$
\begin{gathered}
\dot{\underline{\underline{v}}}_{R, A}=\dot{\underline{\dot{v}}}_{Q, A}+\theta_{B A}\left(\tilde{\omega}_{B A} \theta_{C B} \tilde{\omega}_{b, C}+\theta_{C B} \tilde{\omega}_{C B} \tilde{\omega}_{b, C} \ldots\right. \\
\left.\ldots+\theta_{C B} \dot{\tilde{\omega}}_{b, C}+\theta_{C B} \tilde{\omega}_{b, C} \tilde{\omega}_{D C}\right) \theta_{D C} \underline{r}_{R Q, D}
\end{gathered}
$$

that can be used in Newton's second law

$$
\sum \underline{F}_{A}=m_{b} \underline{\dot{v}}_{R, A}
$$

for the translational motion of the body. The left hand side of (17) can be written as

$$
\sum \underline{F}_{A}=-\theta_{B A} \theta_{C B} \theta_{D C} F_{R, D}+\underline{F}_{G B, A}
$$

and substitution of (18) into (17) and rearranging leads to an expression for the reaction force vector

$$
\underline{F}_{R, D}=\theta_{D C}{ }^{T} \theta_{C B}^{T} \theta_{B A}^{T}\left(\underline{F}_{G B, A}-m_{b} \underline{\dot{\underline{x}}}_{R, A}\right)
$$

Application of Euler's equation on the rotational motion of the body will result in

$$
\sum \underline{M}_{D}=\tilde{\omega}_{D A} J_{b, D} \underline{\omega}_{b, D}+J_{b, D} \underline{\dot{\omega}}_{b, D}
$$

where the left hand side can be written as the sum of all moments

$$
\sum \underline{M}_{D}=-\underline{r}_{R Q, D} \times-\underline{F}_{R, D}-\underline{M}_{R, D}+\underline{M}_{I, D}
$$

acting on point $\mathrm{R}$. Rewriting this last expression leads the reaction moment vector

$$
\begin{gathered}
\underline{M}_{R, D}=\tilde{r}_{R Q, D} \underline{F}_{R, D}+\underline{M}_{I, D}-\tilde{\omega}_{D A} J_{b, D} \underline{\omega}_{b, D} \cdots \\
\ldots-J_{b, D} \underline{\dot{\omega}}_{b, D}
\end{gathered}
$$

where $\tilde{\omega}_{D A}$ and $\underline{\omega}_{b, D}$ in (20) can be written as

$$
\begin{aligned}
& \tilde{\omega}_{D A}=\theta_{D C}^{T}\left(\theta_{C B}^{T} \tilde{\omega}_{B A} \theta_{C B}+\tilde{\omega}_{C B}\right) \theta_{D C}+\tilde{\omega}_{D C} \\
& \underline{\omega}_{b, D}=\theta_{D C} \underline{\omega}_{b, C}
\end{aligned}
$$

\section{SPACAR}

To validate the model derived in this paper, a second independent model is created using SPACAR (Aarts et al., 2008). SPACAR is based on the nonlinear finite element theory for multi-degree of freedom mechanisms and runs in a Matlab environment and capable of analyzing the dynamics of planar and spatial mechanisms and manipulators with flexible links. The code listed below constructs the model of the MEUR in this paper. The SPACAR model consists of a spatial rigid beam element, a spatial wheel element and four spatial hinge elements defined by the commands RBEAM, WHEEL and HINGE. The first number after the command is the element number. The next two or three numbers are the coordinates, where the numbers 1 to 5 are rotational coordinates and 6 to 8 are translational coordinates. The last three numbers represent the initial orientation. 


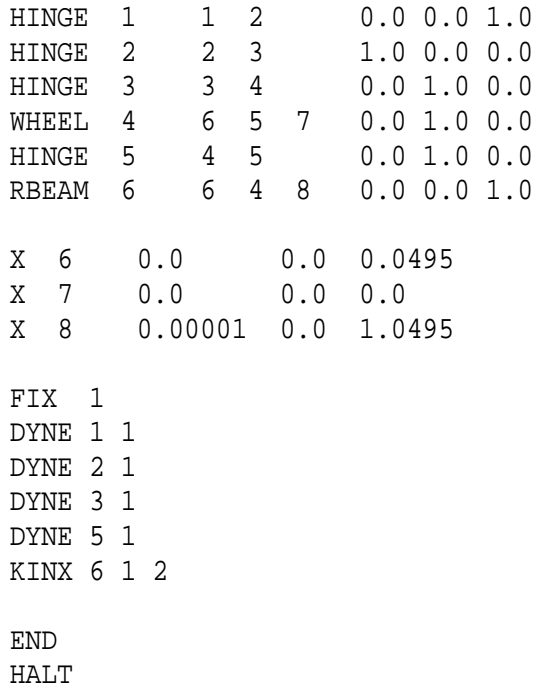

The initial values are defined in the second block by the command $\mathrm{X}$ and assigns the robot dimensions. In the third block the first rotational coordinate is fixed to the world with FIX. DYNE defines the degrees of freedom, being the first deformation of the elements $1,2,3$ and 5, equal to respectively $\psi, \gamma, \theta$ and $\phi$. KINX defines two coordinates where the no-slip condition holds. XM defines the point masses in coordinate 6 and 8 and the inertia's along the $x, y$ and $z$ axis. GRAVITY takes care of the external forces acting on the masses. The third and fourth number of STARTDE defines the initial conditions for $\psi, \gamma, \phi$ and $\theta$ and the initial conditions for their time derivatives. TIMESTEP defines the simulation time followed by the amount of time steps.

\section{SIMULATIONS}

To cross validate the model derived in Section 2 and the model provided by SPACAR in Section 3, time domain simulations are carried out using the numerical values listed in Table 3. During the simulation, the trajectory of the center of the driving wheel is chosen as a measure for the cross validation.

Table 3: Numerical values of MEUR parameters.

\begin{tabular}{|lll|lll|}
\hline \multicolumn{3}{|c|}{ Wheel } & \multicolumn{3}{c|}{ Body } \\
\hline$m_{w}$ & 0.1 & $\mathrm{~kg}$ & $m_{b}$ & 0.2 & $\mathrm{~kg}$ \\
$R$ & 0.0495 & $\mathrm{~m}$ & $d_{r q}$ & 1 & $\mathrm{~m}$ \\
$J_{x x}$ & 0.00002 & $\mathrm{kgm}^{2}$ & $J_{b, x x}$ & 0.8 & $\mathrm{kgm}^{2}$ \\
$J_{y y}$ & 0.00003 & $\mathrm{kgm}^{2}$ & $J_{b, y y}$ & 0.002 & $\mathrm{kgm}^{2}$ \\
$J_{z z}$ & 0.00002 & $\mathrm{kgm}^{2}$ & $J_{b, z z}$ & 0.4 & $\mathrm{kgm}^{2}$ \\
\hline$g$ & 9.81 & $\mathrm{~ms}^{-2}$ & \multicolumn{3}{l}{} \\
\hline
\end{tabular}

Different non-zero initial conditions and constant motor torques are used and listed in Table 4. Time simulations are computed using a non-stiff differential equation solver (Cooper, 2004) and implemented via ode 45 in Matlab. The initial values under \#1 in Table 4 and the definition of the angles in Table 1 are chosen such that the model starts as a stable mechanical system during the simulation. In addition, a small initial roll angle $\gamma=0.6$ is chosen to demonstrate the coupling effects between the lateral and longitudinal motion of the MEUR.

Table 4: Non-zero initial conditions for the simulations.

\begin{tabular}{|l|lll|}
\hline & set \#1 & set \#2 & \\
\hline$\gamma(0)$ & 0.6 & 0.6 & $\mathrm{rad}$ \\
$\varphi(0)$ & $\pi+0.6$ & 0 & $\mathrm{rad}$ \\
\hline$\tau_{1}$ & 0 & 0.1 & $\mathrm{Nm}$ \\
$\tau_{2}$ & 0 & 0 & $\mathrm{Nm}$ \\
\hline
\end{tabular}

It can be observed from the coinciding simulation results depicted in Figure 5 that the time trajectory $(x(t), y(t))$ of the center of the driving wheel starting in point $(0,0)$ undergoes a periodic oscillation in the $x$ direction due to the initial non-zero body pitch angle $\varphi(0)=\pi+0.6$. Interesting to see is also the small motion in the $y$ direction of the center of the driving wheel due to the a initial roll angle $\gamma(0)=0.6$, causing a small change in yaw angle $\psi(t)$. With simulation results of the body pitch angle $\varphi(0)=0$ and the roll angle $\gamma(0)=0.6$ depicted in Figure 6, the MEUR model now starts in upward and unstable direction and during the simulation we assume the MEUR can fall and oscillate through the base plane. In addition, a constant torque $\tau_{1}=0.1$ is applied between the driving wheel and body. In Figure 6, both models follow the same trajectories very closely for some time but diverge eventually. This is due to the fact that the model of the mechanical system is unstable at the initial condition, and small numerical errors in either initial conditions or numerical integration leads to exponentially 
increasing differences in the simulation.

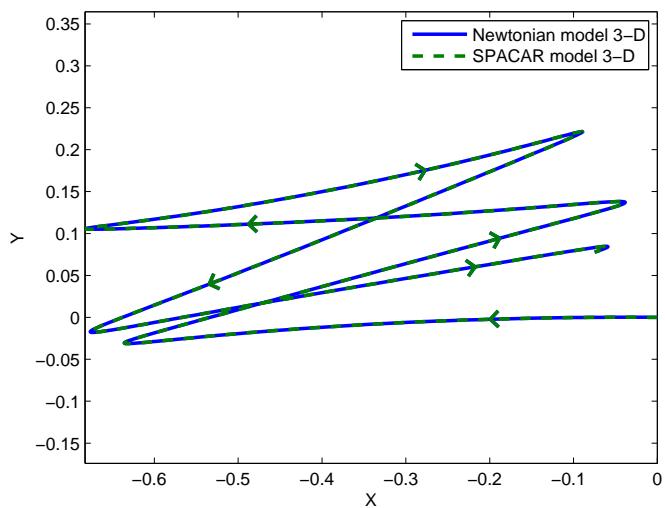

Figure 5: Simulation results of the trajectory of the center of the driving wheel starting in point $(0,0)$ using the parameters of set \#1 in Table 4 .

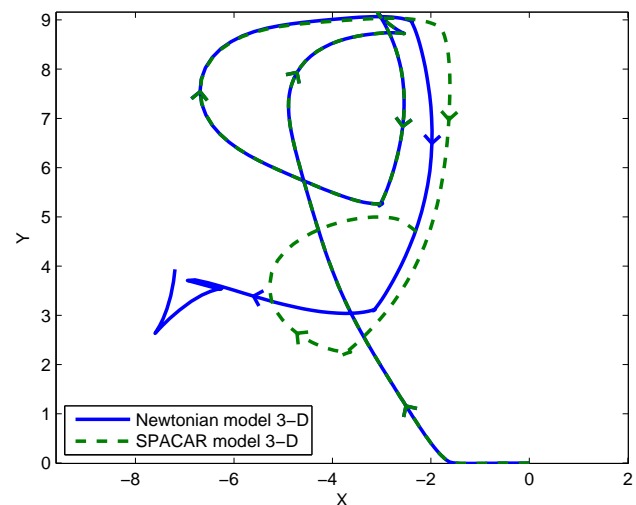

Figure 6: Simulation results of the trajectory of the center of the driving wheel starting in point $(0,0)$ using the parameters of set \#2 in Table 4 .

\section{CONCLUSIONS}

This paper presents a three dimensional nonlinear dynamic model for a Moment Exchange Unicycle Robot (MEUR). The model is derived using both Newtonian mechanics and a non-linear finite element package for multi-degree of freedom mechanisms called SPACAR. The simulation results presented in this paper cross validate the Newtonian and the SPACAR model, as simulations of the center of the driving wheel coincide. Differences is simulations attributed to small errors in the in either initial conditions or numerical integration can only be observed in case of an unstable initial condition. In addition, the simulation results demonstrate the coupling between lateral and longitudinal motion the center of the driving wheel. Coupling effects are small only in the case of limited (stabilized) motions of the MEUR.

\section{REFERENCES}

Aarts, R., Meijaard, J., and Jonker, J. (2008). SPACAR User Manual.

$\mathrm{Au}, \mathrm{K}$. and Xu, Y. (1999). Decoupled dynamics and stabilization of single wheel robot. In IEEE/RSJ Int. Conf. on Intelligent Robots and Systems, volume 1, pages 197-203.

Cooper, J. (2004). An Introduction to Ordinary Differential Equations. Cambridge University Press.

Dao, M. and Liu, K. (2005). Gain-scheduled stabilization control of a unicycle robot. JSME International Journal Series C: Mechanical Systems, Machine Elements and manufacturing, 48(4):649-656.

Hughes, P. (1986). Spacecraft Attitude Dynamics. J. Wiley.

Kolve, D. (1993). Describing an attitude. In Proc. Annual Rocky Mountain Guidance and Control Conference, volume 93, pages 289-303.

Majima, S., Kasai, T., and Kadohara, T. (2006). A design of a control method for changing yaw direction of an underacuatted unicycle robot. In Proc. TENCON 2006, IEEE Region 10 Conference, pages 1-4.

Nakajima, R., Tsubouchi, T., Yuta, S., and Koyanagi, E. (1997). A development of a new mechanism of an autonomous unicycle. In IEEE/RSJ Int. Conf. on Intelligent Robots and Systems, volume 2, pages 906-912.

Naveh, Y., Bar-Yoseph, P., and Halevi, Y. (1999). Nonlinear modeling and control of a unicycle. Dynamics and Control, 9:279-296.

Ohsaki, H., Kinoshita, M., Sugimoto, Y., Yoshida, K., Iwase, M., and Hatakeyama, S. (2008). Development of a control system for supporting to grow human skill of a unicycle. In SICE Annual Conference, pages 2229-2233.

Schoonwinkel, A. (1987). Design and Test of a Computer Stabilized Unicycle. PhD thesis, Stanford Univ., CA.

Sheng, Z. and Yamafuji, K. (1997). Postural stability of a human riding a unicycle and its emulationby a robot. IEEE Transactions on Robotics and Automation, 13:709-720.

van Pommeren, J. (2007). The unibot, design and control of a self-balancing unicycle robot. Master's thesis, University of Twente.

Vos, D. and Flotow, A. V. (1990). Dynamics and nonlinear adaptive control of an autonomous unicycle: Theory and experiment. Proc. of the 29th IEEE Conf. on Decision and Control, 1:182-187.

Zenkov, D., Bloch, A., and Marsden, J. (1999). Stabilization of the unicycle with rider. In Proc. 38th IEEE Conf. on Decision and Control, volume 4, pages $3470-3471$. 